\begin{tabular}{|c|l|}
\hline Title & Electromagnetic and thermal simulation of stacked NI REBCO pancake coils after normal-state transition \\
\hline Author(s) & Miyao, Ryosuke; Igarashi, Hajime; Noguchi, So \\
\hline Citation & $\begin{array}{l}\text { International journal of applied electromagnetics and mechanics, 59(1), 181-189 } \\
\text { https://doi.org/L0.3233/AAE-171219 }\end{array}$ \\
\hline Issue Date & 2019-03-21 \\
\hline Doc URL & http://hdl.handle.net/2115/73872 \\
\hline Rights & The final publication is available at IOS Press through http://dx.doi.org/10.3233/AAE-171219 \\
\hline Type & article (author version) \\
\hline File Information & ISEMPaper._r2.pdf \\
\hline
\end{tabular}

Instructions for use 


\title{
Electromagnetic and Thermal Simulation of Stacked NI REBCO Pancake Coils after Normal-State Transition
}

\author{
Ryosuke MIYAO ${ }^{\mathrm{a}}$, Hajime IGARASHI ${ }^{\mathrm{a}}$ and So NOGUCHI ${ }^{\mathrm{a}, \mathrm{b}}$ \\ ${ }^{a}$ Graduate School of Information Science and Technology, Hokkaido University, Sapporo 060-0814, \\ Japan \\ b Plasma Fusion and Science Center, Massachusetts Institute of Technology, Cambridge, MA 02139, \\ USA
}

\begin{abstract}
This paper presents the current and thermal phenomena of multi-stacked No-Insulation (NI) $\mathrm{REBa}_{2} \mathrm{Cu}_{3} \mathrm{O}_{7-x}$ (REBCO, RE = Rare Earth) pancake coils during a local normal-state transition. An NI winding technique is expected to enhance the thermal stability of REBCO pancake coils, and it was verified in numerical simulations on a single pancake coil. However, a multi-stack of pancake coils are used to generate a high magnetic field in practical applications. When a normalstate transition occurs in one of multi-stacked pancake coils, it may deteriorate the stability of the other pancake coils. Therefore, we have developed a numerical simulation code to clarify the current and thermal behaviors of multi-stacked NI REBCO pancake coils using a partial element equivalent circuit model and a thermal finite element method.
\end{abstract}

Keywords: High Temperature Superconducting Magnet, PEEC method, No-Insulation REBCO Pancake Coil.

\section{Introduction}

In recent years, a $\mathrm{REBa}_{2} \mathrm{Cu}_{3} \mathrm{O}_{7-x}(\mathrm{REBCO}, \mathrm{RE}=$ Rare Earth) pancake coil using a No-Insulation (NI) winding technique $[1,2]$ has received attention to realize REBCO applications. In a conventional REBCO pancake coil, a sheet of insulation is co-wound between turns. However, in an NI REBCO pancake coil, an insulator is removed, and a current can flow between turns. Therefore, when a local normal-state transition occurs in an NI pancake coil due to a degradation of REBCO tape or a temperature rising, a current can also flow from a turn to adjacent turns so as to avoid the local normal-state zone (local hot spot) as seen in Fig. 1. Thus, the NI winding technique makes it possible to prevent a current from flowing into highly resistive REBCO region, and it is expected to enhance the thermal stability. The NI REBCO magnet stability is so high that burning-out does not occur even when a pancake coil quenches. Moreover, as a REBCO pancake coil does not even burn out, it can be reused after the coil is cooled again. Due to the stability enhancement, NI REBCO pancake coils are expected to be applied to NMR/MRI and accelerator $[3,4]$.
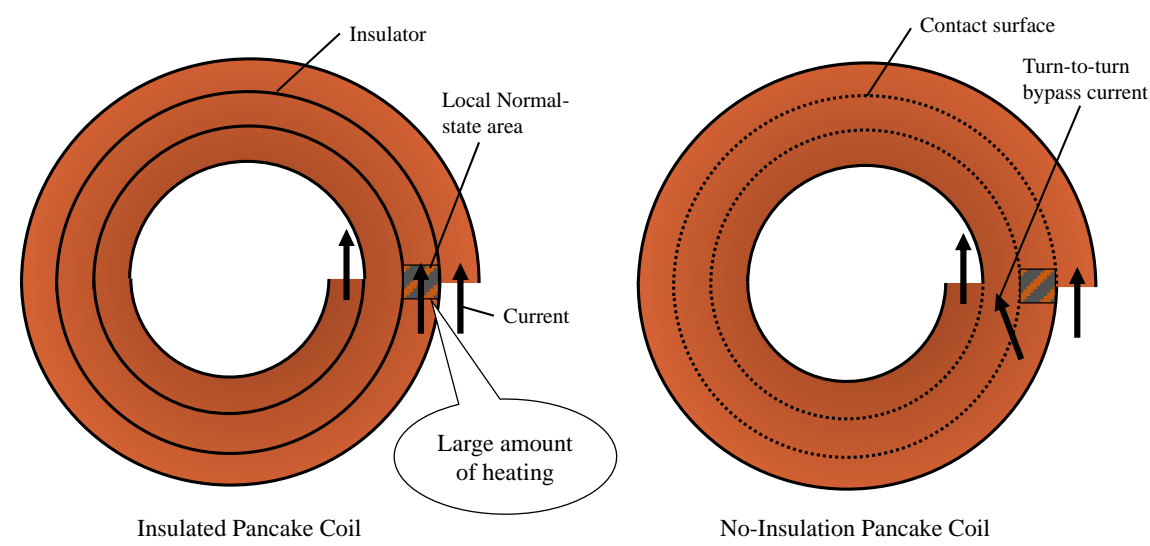

Fig. 1. Schematic drawings of current behavior in conventional insulated pancake coil and newly developed No-Insulation pancake coil. Figures indicate the top view of single pancake coils having 3 turns. 
The high stability of NI REBCO pancake coils have been verified in experiments, however it was shown only with an overcurrent test [1]. Based on this background, we have previously developed the numerical simulation to confirm the current and thermal behaviors of a single NI REBCO pancake coil on a local normal-state transition using a partial element equivalent circuit (PEEC) method [5, 6]. However, in real applications, multi pancake coils are usually stacked to generate a high magnetic field. When one of stacked pancake coils transitions into a normal state, it is confirmed that the normal-state-transitioned pancake coil loses a generation of self-magnetic field [5], and a current is induced in the other pancake coils. The induced current is consumed as Joule heating generation in the pancake coils, and the same amount as the magnetic energy stored in the quenched coil is consumed by the contact resistance. Consequently, the Joule heating generation, i.e. temperature rising, may deteriorate the stability of the not-quenched pancake coils. In fact, it was observed in experiments that the quench of one pancake coil sequentially propagated to the other pancake coils. Especially at low operating temperature (i.e. a large operating current), the stored magnetic energy is so large that the Joule heating invokes to burn out pancake coils. Thus, in order to confirm whether the quench propagation invokes the burning-out or not, the behavior of multi-stacked NI REBCO pancake coils must be investigated in simulation in detail. Therefore, we have previously developed an electromagnetic simulation for multi-stacked NI REBCO pancake coils [7]. However, the developed simulation code cannot reproduce quench propagation because the thermal behavior is not taken into account. Consequently, we have newly extended the previously developed method so that a thermal analysis can be performed by 2-D finite element method (FEM). In this paper, we present the simulation method of multi-stacked NI REBCO double pancake coils on local normal-state transition combining the PEEC method and the 2-D FEM. We also present the simulation results of therestacked NI double pancake coils after the quench, with comparison to the results of the conventional method [7].

\section{Simulation Method}

With the proposed simulation method, the electromagnetic and thermal behaviors of multi-stacked NI REBCO pancake coil are simulated. Fig. 2 shows the flowchart of the developed simulation method. First of all, the electromagnetic behavior is computed by the PEEC method. It derives the current distribution inside each pancake coil and the Joule heating generation as an input of the thermal analysis. Then, the thermal analysis is performed by the 2-D FEM, inputting the obtained heating parameters. It derives the temperature distribution inside of each pancake coil, and the temperature determines the equivalent electrical resistivity of REBCO layer at the next time step. This is the outline of the proposed simulation method.

In the circuit analysis, an NI pancake coil is subdivided to partial elements in circumferential direction every turn, and they are expressed as an equivalent circuit. Fig. 3 shows the cross section of REBCO tape and the schematic drawing of the equivalent circuit model of one partial element. The equivalent circuit of the partial element consists of the equivalent resistance of superconducting layer, the copper stabilizer resistance, the self-inductance, and the mutual inductances with the other elements. The Hastelloy substrate is not considered in the circuit analysis since it has a large value of resistance. The equivalent resistance of superconducting layer is calculated according to the $n$-value model, and

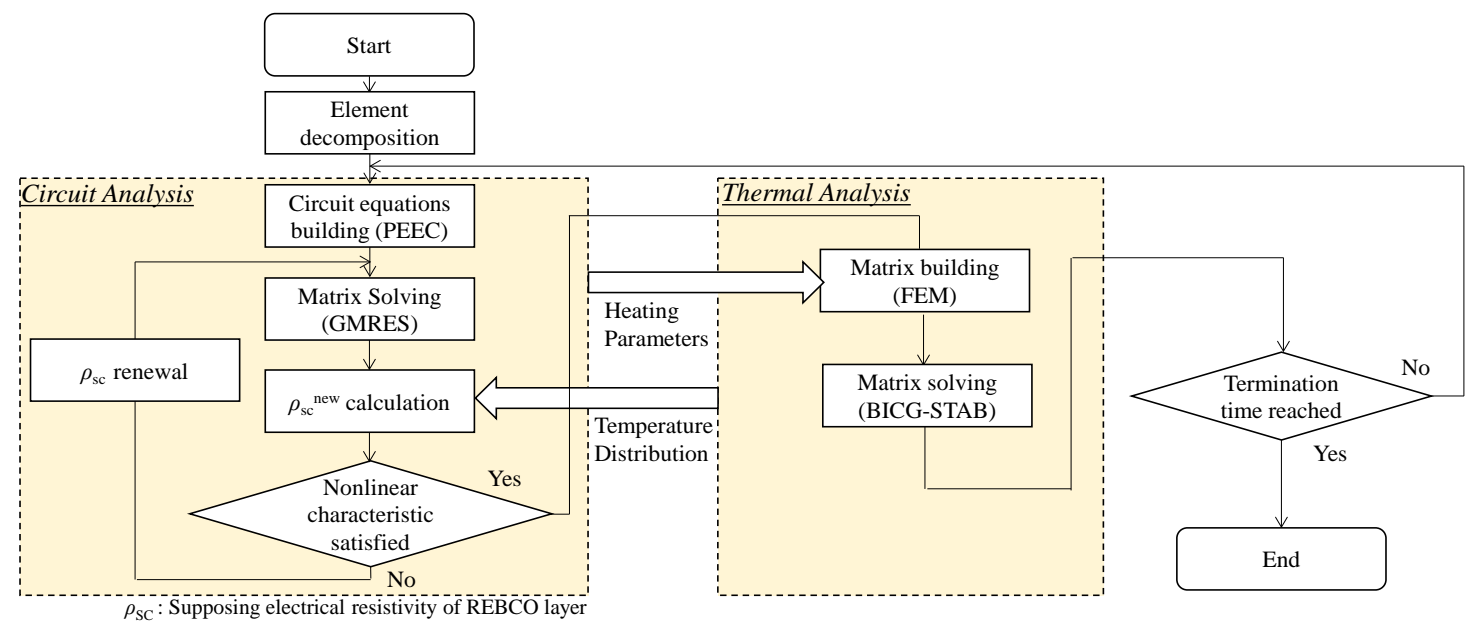

Fig. 2. Flowchart of the newly developed simulation coupling with the PEEC method and the 2-D thermal FEM. 


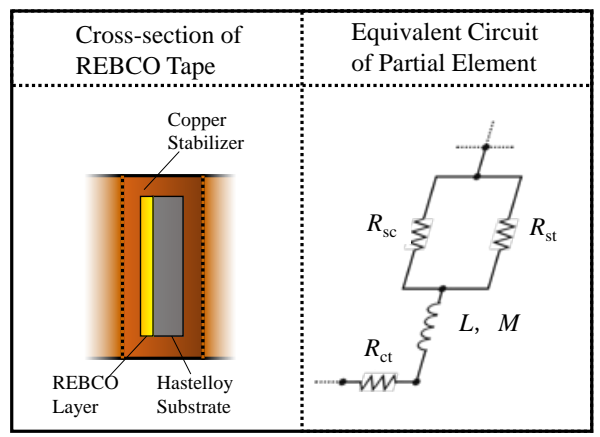

Fig. 3. Cross section of REBCO tape and schematic drawing of equivalent circuit model of one partial element. Equivalent circuit of partial element consists of the equivalent resistance of superconducting layer $R_{\mathrm{SC}}$, the copper stabilizer resistance $R_{\text {st, }}$, the self-inductance $L$, and the mutual inductances $M$.

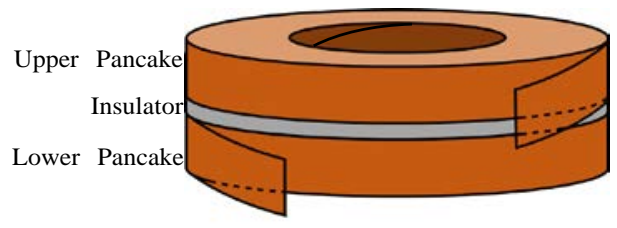

Double pancake coil

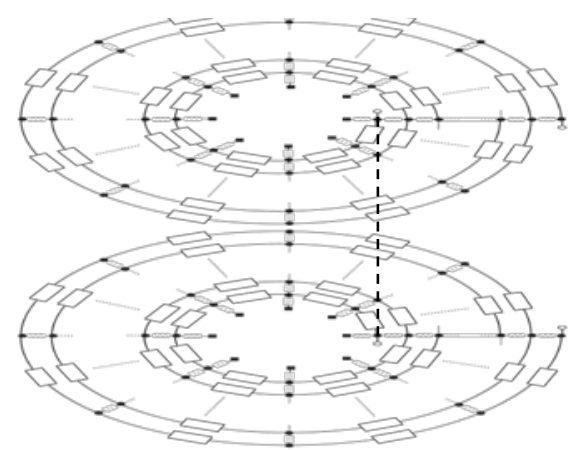

PEEC model of double pancake coil

Fig. 4. F.ntire view of enuivalent circuit of NI RF.BCO double nancake coil

the critical current density is obtained from an approximate equation derived from the experimental data [8]. Since there is no insulation between turns, the contact resistance has to be considered in the radial direction. The entire view of equivalent circuit of NI REBCO double pancake coil is shown in Fig. 4. It is expressed as two single pancake coils connecting at the innermost turn with a crossover turn. Each single pancake coil is electrically and thermally insulated each other, however the current and the heat flow into an adjacent single pancake coil through a crossover turn. The equivalent circuit model is computed from the equations based on the Kirchhoff's first and second laws as follows:

$$
\begin{aligned}
& I_{\mathrm{L}, i}-I_{\mathrm{L}, i-1}+I_{\mathrm{ct}, i}-I_{\mathrm{ct}, i-d}=0 \quad\left(I_{0}=I_{\mathrm{op}}, I_{n+1}=I_{\mathrm{op}}\right) \\
& \sum_{j=i}^{i-d-1}\left(\sum_{k=1}^{n} M_{j k} \frac{I_{\mathrm{L}, k}-I_{\mathrm{L}, k}^{\prime}}{\Delta t}+I_{\mathrm{L}, j} \frac{R_{\mathrm{sc}, j} R_{\mathrm{st}, j}}{R_{\mathrm{sc}, j}+R_{\mathrm{st}, j}}\right)-I_{\mathrm{ct}, i} R_{\mathrm{ct}, i}=0
\end{aligned}
$$

where, $i, d, \Delta t$, and $n$ are the element number, the number of circumferential division, the time step of simulation, and the number of elements, respectively. $I_{\mathrm{L}}$ and $I_{\mathrm{ct}}$ are, respectively, the currents flowing in the circumferential and radial directions. $I^{\prime}{ }_{\mathrm{L}}$ means the circumferential current one time step before. $R_{\mathrm{sc}}, R_{\mathrm{st}}$, and $R_{\mathrm{ct}}$ are the equivalent resistance of REBCO layer, the resistance of copper stabilizer, and the contact resistance between turn-to-turn windings, respectively. $M_{i j}$ is the self $(i=j)$ and mutual $(i \neq j)$ inductances of the partial elements.

After the circuit analysis, the thermal analysis is performed by 2-D FEM. The governing equation is given by

$$
\rho c \frac{\partial T}{\partial t}=\lambda \Delta T+Q
$$

where, $\rho, c, T, t, \lambda$, and $Q$ are the specific heat, the mass density, the temperature, the time, the thermal conductivity, and the heat density, respectively. The heat propagates in the radial and circumferential directions. The heat density is obtained from the circuit analysis result. The pancake-topancake heat propagates only through a crossover turn. An adiabatic condition is supposed as a cooling condition, and the specific heat and the thermal conductor are temperature-dependent that are decided from the value of the previous time step. The thermal analysis produces the temperature distribution necessary to compute the critical current density.

\section{Simulation Model and Result}

In this section, we present a simulation result of multi-stacked NI pancake coils after quench. Table 1 lists the specifications of the simulated NI REBCO magnet. The magnet consists of three-stacked NI 
REBCO double pancake coils. The every pancake coil has 100 turns, and they are wound with SuperPower 2G-HTS tape [9] using the NI winding technique. The turn-to-turn contact resistivity is supposed to be $7.0 \mu \Omega \mathrm{cm}^{2}$ [10]. The simulation condition is listed in Table 2. The magnet is operated at $10 \mathrm{~K}$. The coil critical current is $860 \mathrm{~A}$, and the magnet is operated with $100 \%$ load factor. It is supposed that the entire lowermost pancake coil transitions into a normal state in the simulation.

The simulation results by the proposed method are presented below, together with comparison of the conventional method in which a thermal behavior is not considered.

TABLE 1

SPECIFICATIONS OF SIMULATION NI REBCO MAGNET

\begin{tabular}{|c|c|}
\hline Parameters & Values \\
\hline \multicolumn{2}{|l|}{ REBCO tape } \\
\hline REBCO tape width [mm] & 4.0 \\
\hline REBCO tape Thickness [mm] & 0.096 \\
\hline Copper stabilizer thickness $[\mu \mathrm{m}]$ & 20.0 \\
\hline REBCO layer thickness $[\mu \mathrm{m}]$ & 1.0 \\
\hline$I_{\mathrm{c}} @ 77 \mathrm{~K}$, self-field [A] & 115.0 \\
\hline \multicolumn{2}{|c|}{ Single pancake coil } \\
\hline Coil i.d, o.d [mm] & $60.0,79.2$ \\
\hline Flange (insulator) width [mm] & 1.0 \\
\hline Number of turns (each pancake) & 100 \\
\hline Contact resistivity $\left[\mu \Omega \cdot \mathrm{cm}^{2}\right]$ & 70.0 \\
\hline \multicolumn{2}{|l|}{ Magnet } \\
\hline Number of double pancakes & 3 \\
\hline Coil height [mm] & 29 \\
\hline Inductance $[\mathrm{mH}]$ & 23.9 \\
\hline
\end{tabular}

TABLE 2

SiMULATION CONDITIONS

\begin{tabular}{ll}
\hline \hline Parameters & Values \\
\hline \hline Time step [s] & 0.1 \\
Simulation time [s] & 50.0 \\
Load factor [\%] & 100 \\
Operating temperature [K] & 10 \\
\hline
\end{tabular}

\subsection{Current and Temperature Distributions}

Fig. 5 shows the circumferential current distribution at $1.0 \mathrm{~s}$ after the normal-state transition of (a) the conventional method and (b) the proposed method. In the both figures, the circumferential current of the lowermost pancake coil transitioned into a normal state is almost zero, because almost the current flows in the radial direction. Meanwhile, the different distribution can be seen in the other notquenched coils. In Fig. 5 (a), the circumferential current in \#2-U-\#3-U coils is larger than an operation current of $860 \mathrm{~A}$. This is due to the induced current by the quench of the lowermost pancake coil. However, in Fig. 5 (b), the current decreasing is observed in \#2-L and \#3-U. This is because these coils quenched due to the induced current.

Next, the temperature distribution is shown in Fig. 6. In the pancake coils where the current decreasing occurs, the temperature rises up. This temperature rising causes the critical current decreasing. Subsequently, the current flows in the radial direction. As shown above, the proposed

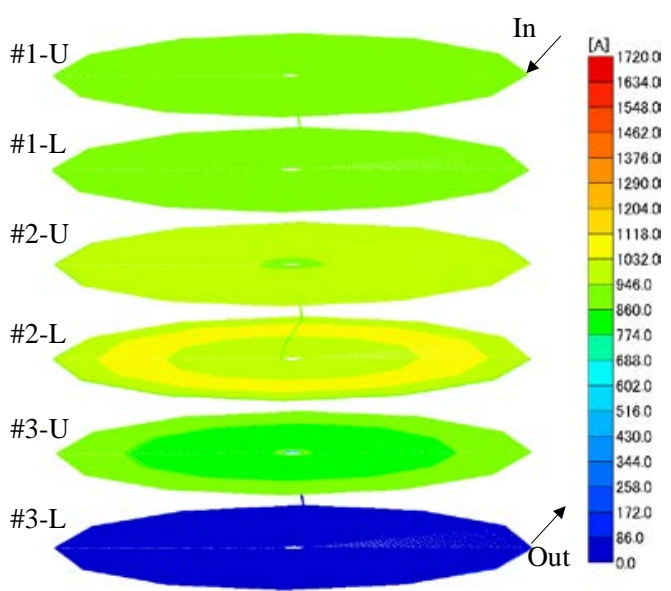

(a)

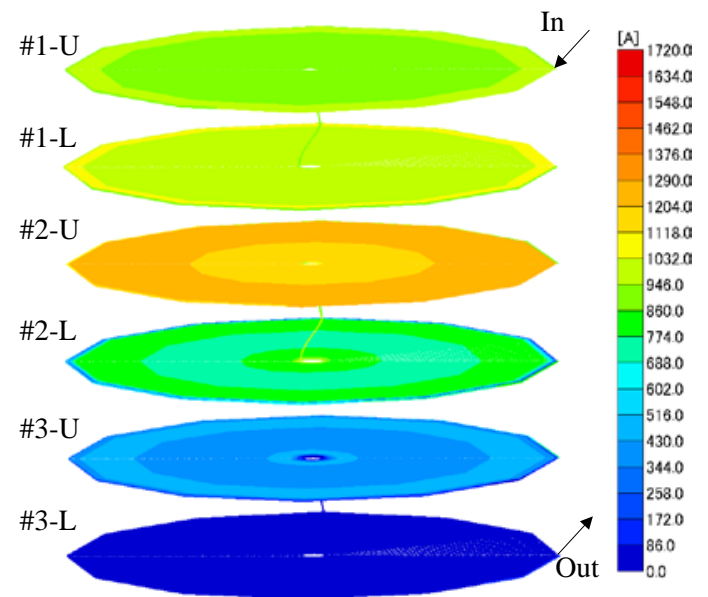

(b)

Fig. 5. Circumferential current distribution at $1.0 \mathrm{~s}$ after normal-state transition by (a) conventional method (not including thermal analysis) and (b) proposed method (including thermal analysis) 
method makes it possible to reproduce sequential quench propagation from pancake to pancake.

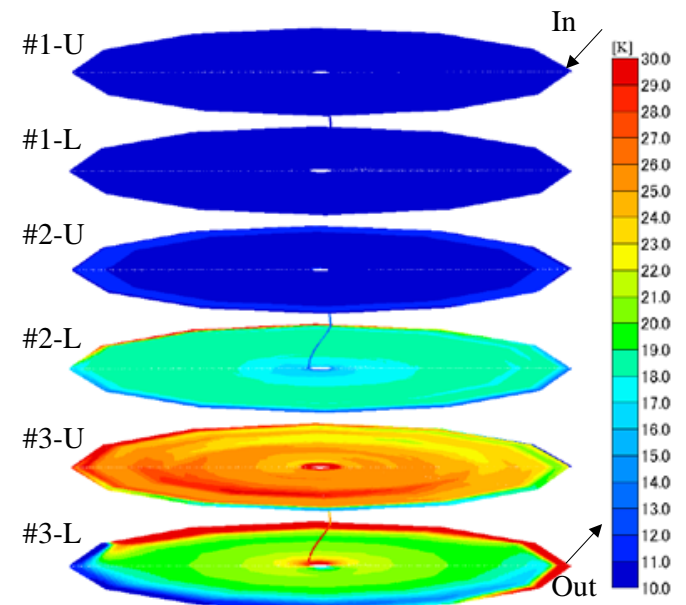

Fig. 6.Temperature distribution at $1.0 \mathrm{~s}$ after normal-state transition by proposed method

\subsection{Time Transition of Current and Temperature}

In this section, the time transitions of the current and temperature are presented. Fig. 7 shows the average circumferential current transition of each pancake by (a) the conventional method and (b) the proposed method. In order to except the influence of heat propagation from the crossover turn, the average current is calculated only by the outer 50 turns. In Fig. 7 (a), the circumferential current of every pancake coil increases after the normal-state transition of \#3-L. Although the currents gradually decrease, they do not go below the operating current. Meanwhile, in Fig. 7 (b), though the currents increases temporarily, it is immediately decreases to almost $0 \mathrm{~A}$.

Next, the average temperature transition of each pancake is shown in Fig. 8. At the same time of the current increasing, the temperature also increases. By the temperature rise, the critical current decreases. Subsequently, the more heating is followed, and the circumferential currents decrease. The circumferential current decreasing (i.e. magnetic field decreasing) anew leads to quench the other pancake coil. Consequently, all of the pancake coils will quench sequentially. This is the mechanism of the sequential quench propagation from pancake to pancake. As shown above, the proposed simulation method makes it possible to clarify the mechanism of quench propagation by combining a thermal analysis.

As mentioned above, we showed the possibility of sequential quench propagation. However, even when the sequential quench occurs, the NI pancake coils do not receive any damage, and they can be reused as long as they do not burn out. In Fig. 8, the average temperature of the \#3-L of turn-to-turninsulated coils is also shown with a black dotted line. When the pancake coil is insulated between turns, the temperature drastically increases, and it reaches to $500 \mathrm{~K}$ immediately. Meanwhile, in the NI pancake coil, the temperature is not so high that a coil burns out. As the result, we can conclude that the NI winding technique can keep the coils healthy even when sequential quench propagation occurs.

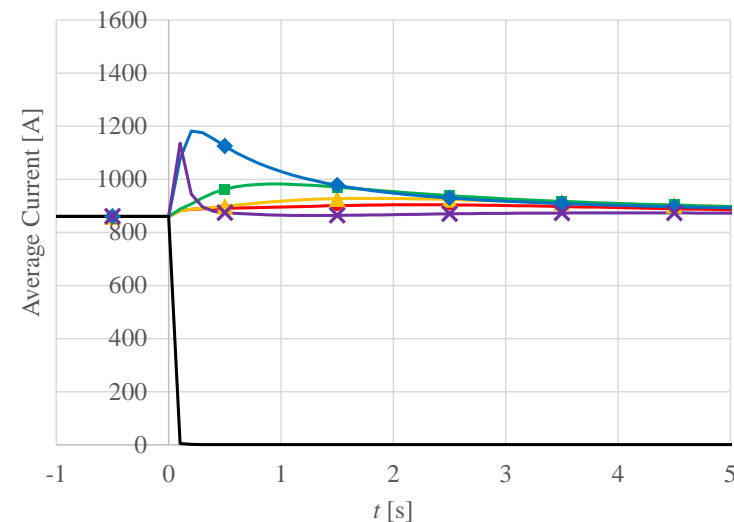

(a)

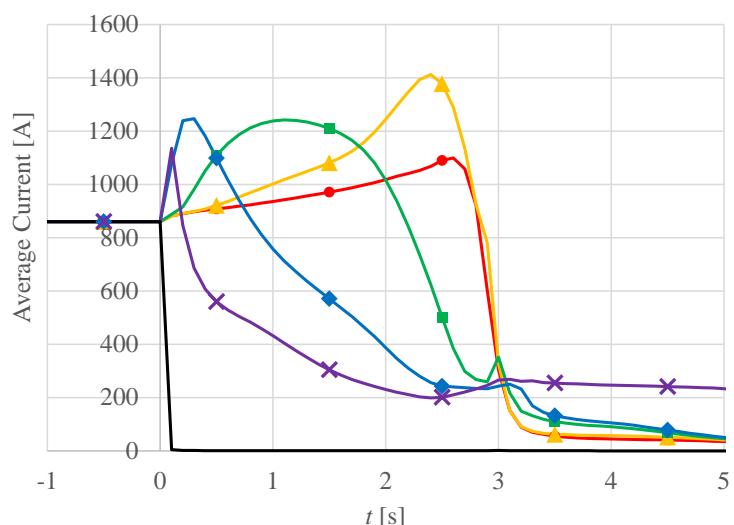

(b)

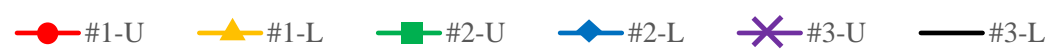

Fig. 7. Average current transition of each pancake by (a) conventional method and (b) proposed method. In order to except influence of heat propagation from crossover turn, average current is calculated only by outer 50 turns. 


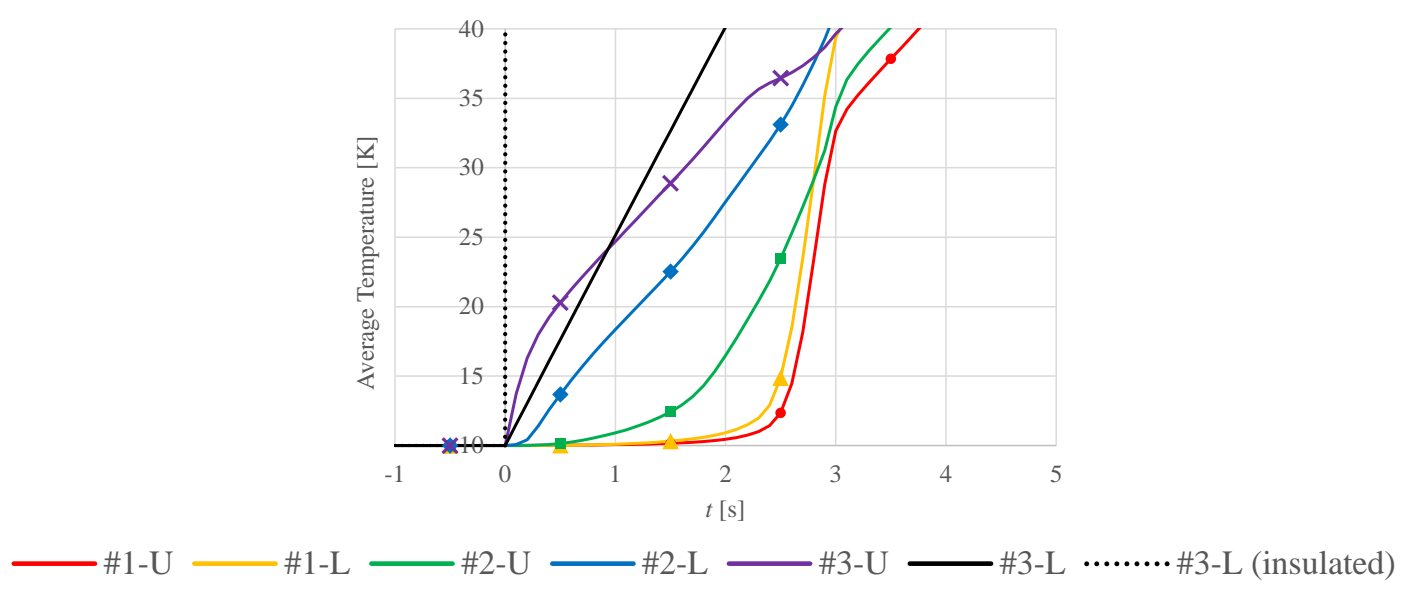

Fig. 8. Average temperature transition of each pancake. In order to except influence of heat propagation from crossover turn, average current is calculated only by outer 50 turns. Black-dotted line is the average temperature of \#3-L which is the lowermost coil of three-stacked insulated REBCO coils.

\section{Conclusion}

In this paper, we present a new numerical simulation method to investigate the thermal and electromagnetic behaviors of multi-stacked NI REBCO pancake coils. In the simulation, the circuit analysis is performed by the PEEC method, and the thermal analysis is performed by the 2-D FEM. The circuit analysis identifies the current distribution and the Joule heat generation. Using the obtained parameters, the thermal analysis is performed to obtain the temperature distribution. The temperature distribution decides the critical current of REBCO tape in the circuit analysis.

We also present the simulation result of three-stacked NI REBCO pancake coils after the normalstate transition in the lowest pancake coil, by the conventional method (not including the thermal analysis) and the proposed method, individually. In the conventional method, the current reduction is confirmed only in the pancake coil which firstly transitioned into a normal-state transition. However, in the proposed method, the current reduction is confirmed in the other pancakes and the quench propagation through the entire magnet is reproduced.

In the simulated magnet, it is concluded that the temperature does not rise up to a terrible temperature to burn out coils, and the stability can be maintained. However, since the radius of simulated magnet is small, we will investigate whether a larger-size magnet using NI winding technique, which has a large inductance and a large stored magnetic energy, is damaged by a sequential quench or not.

\section{References}

[1] S. Hahn, D. K. Park, J. Bascuñán, and Y. Iwasa, "HTS pancake coils without turn-to-turn insulation,” IEEE Trans. Appl. Supercond., vol. 21, no. 3, pp. 1592-1595, Jun. 2011.

[2] S. B. Kim, A. Saito, T. Kaneko, J. H. Joo, J. M. Jo, Y. J. Han, and H.S. Jeong, "The characteristics of the normal-zone propagation of the HTS coils with inserted Cu tape instead of electrical insulation,” IEEE Trans. Appl. Supercond., vol. 22, no. 3, Jun. 2012, Art. ID. 4701504.

[3] Y. Iwasa, J. Bascunan, S. Hahn, J. Voccio, Y. Kim, T. Lecrevisse, J. Song, and K. Kajikawa, "A high-resolution 1.3GHz/54-mm LTS/HTS NMR magnet,” IEEE Trans. Appl. Supercond., vol. 25, no. 3, Jun. 2015, Art. ID. 4301205.

[4] K. L. Kim, J. B. Song, Y. H. Choi, D. G. Yang, and H. Lee, "Feasibility Study of a No-Insulation 1.5-T/600-mm AllREBCO Magnet for MRI Systems,” IEEE Trans. Appl, Supercond., vol. 27, no. 4, Jun. 2017, Art. 4600204.

[5] T. Wang, S. Noguchi, X. Wang, I. Arakawa, K. Minami, K. Monma, A. Ishiyama, S. Hahn, and Yukikazu Iwasa, “Analyses of Transient Behaviors of No-Insulation REBCO Pancake Coils During Sudden Discharging and Overcurrent," IEEE Trans. Appl. Supercond., vol. 25, no. 3, Jun. 2015, Art. ID. 4603409

[6] T. Oki, A. Ikeda, T. Wang, A. Ishiyama, S. Noguchi, K. Monma, T. Watanabe, and S. Nagaya, "Evaluation on Quench Protection for No-Insulation REBCO Pancake Coil,” IEEE Trans. Appl. Supercond., vol. 26, no. 4, Jun. 2016, Art. ID. 4702905.

[7] S. Noguchi, et al., "Current Behavior Simulation in Stacked NI REBCO Pancake Coils During Local Normal-State Transition,” IEEE Trans. Appl. Supercond., vol. 27, no. 4, Jun. 2017, Art. ID. 4603205.

[8] H.Ueda, et al., "Numerical Simulation on Magnetic Field Generated by Screening Current in 10-T-Class REBCO Coil," IEEE Trans. Appl. Supercond., vol. 26, no. 4, pp. 1-5, Jun. 2016.

[9] http://www.superpower-inc.com/content/2g-htswire

[10] X. Wang et al., "Turn-to-turn contact characteristics for an equivalent circuit model of no-insulation ReBCO pancake coil,” Supercond. Sci. Technol., vol. 26, no. 3, Mar. 2013, Art. no. 035012. 